\title{
Mathematical Modeling of Discharge Plasma Generation and Diffusion Saturation of Metals and Alloys
}

\author{
Nguyen Bao Hung ${ }^{1}$, T. V. Koval ${ }^{2}$, Tran My Kim An ${ }^{3}$ \\ ${ }^{1,2,3}$ National Research Tomsk Polytechnic University \\ Tomsk, Russia \\ E-mail: ${ }^{1}$ baohung.ng@gmail.com, ${ }^{2}$ tvkoval@mail.ru, ${ }^{3}$ tranmykiman@gmail.com
}

\begin{abstract}
This paper presents a mathematical modeling of the plasma generation in a hollow cathode and the diffusion saturation of metals and alloys by nitrogen atoms in the plasma of a low-pressure non-self-sustained glow discharge. Characteristics of gas discharge are obtained. The relations between main technological parameters and structural-phase states of nitrited material are defined. Furthermore, this paper leads a comparison of calculated results with the experimental data.
\end{abstract}

Keywords: hollow cathode, glow discharge, low-pressure discharge, plasma, plasma cathode, plasma nitriding, diffusion

\section{INTRODUCTION}

One of the most effective methods of improving operational properties of machine parts and mechanisms is the use of materials with special properties of the surface layer modified by various methods. It is more profitable than to modify the entire volume of material. Among the different methods to improve lifetime and reliability of parts, ion-plasma surface treatment in the gas discharges plasma and, in particular, nitriding have been widely developed $[1,2]$ in recent years.

A target for plasma treatment is immersed in gas plasma in which ions are accelerated toward the target in the electric double layer formed between the target and discharge column. The saturating element (gas atoms) in the treated target volume propagates due to heat-activated diffusion whose rate depends mostly on the treatment temperature and saturating element gradient. The main parameters of plasma treatment are the gas composition or gas mixture ratio, temperature and time of the process, operating pressure, discharge parameters, dissociation and ionization degrees of the working gas, ion energy, and ion current density at the surface of a treated target [1]. Most of these parameters are interrelated. Therefore, the gas composition or gas mixture ratio affects the discharge initiating voltage and hence the ion energy, and the voltage and current density are limited by the temperature allowable for treatment and by the gas pressure. Nitriding at $260-510^{\circ} \mathrm{C}$ provides the formation of hard nitride layers of thickness ranging up to $200-300 \mu \mathrm{m}[4,5]$.

The vacuum plasma technology used for surface modification of materials and large-size objects is based on the generation of low-temperature glow discharge plasmas [4-9] for which it is required to provide an ion current density of $\sim 1 \mathrm{~mA} / \mathrm{cm}^{2}$ to a treated surface and a discharge operating voltage of hundred volts [8-11].

The complexity and multiple relations of nitriding process make difficult to define the general laws in the structuration of modified layers and their properties [12-16]. Such problems can be solved by mathematical modeling of the processes in plasma generation and material nitriding. This is also an effective way to establish the mechanisms of forming the modified layer with specified properties and their dependences on technological parameters (pressure and gas kind, current and discharge voltage, ion current density) of processes.

It assumes that an exact quantitative relation between the parameters of processes with structural-phase states and with the diffusion mechanism of nitrogen atoms will optimize the existing methods of nitriding and herewith allows developing new modification modes based on currently available data.

The aim of this work is to develop a mathematical model of the plasma generation in a hollow cathode and the diffusion saturation of metals and alloys by nitrogen atoms in the plasma of a low-pressure non-self-sustained glow discharge. This work seeks to obtain discharge gas characteristics and determine the relations between main technological factors and diffusion saturation of nitrided metals and alloys by nitrogen atoms. In this work the results of mathematical modeling are compared with the experimental data of steel nitriding.

\section{MOdeling OF GAS Discharge IN THE Hollow CATHODE}

The cylindrical hollow cathode used in experiments on plasma generation is shown in Fig. $1[8,9]$. Anode 1 in the form of two tubes of total area $S_{a}$ is located at the side wall of the cathode. The anode area $S_{a}$ is varied by displacing the anode deep into the cathode cavity the volume of which is $V_{c}=2 \times 10^{5} \mathrm{~cm}^{3}, S_{a}=200 \ldots 500 \mathrm{~cm}^{2}$. The plasma is generated both in the main (self-sustained) mode and in the mode in which the discharge is sustained by an electron beam extracted from the plasma of auxiliary arc discharge 3. The plasma is formed inside the volume of the hollow cathode. The cathode potential fall $U_{c}$ is almost equal to the discharge voltage $U$ : $U_{c}=U-U_{a} \approx U$, where $U_{a}$ is the negative anode potential fall. The plasma ions accelerated in the cathode layer provides ion- 
electron emission from the cathode surface. The electrons accelerated and set in oscillatory motion in the hollow cathode (with about the same probability of ionization at any point of the cathode cavity) lose their energy in collisions with neutrals and ensure a self-sustained discharge. This provides high homogeneity of the ion current density, which is defined by the properties of the discharge plasma.

The hollow-cathode discharge is described by equations of energy balance of charged particles and current continuity which allow us to relate the internal parameters (temperature, plasma potential) to the external parameters (working gas pressure, discharge current and voltage, system geometry). From the balance equation for fast electrons, we can obtain the discharge operating condition. In the general case of auxiliary discharge current injection $I_{\text {ext }}$, the balance equation for fast electrons is written in the following form:

$$
\frac{\gamma I_{i}}{e}+\frac{\delta I_{i}}{e}=\frac{n_{f} V v_{i}}{u}+\frac{S_{a}}{4} n_{f}\langle\mathrm{v}\rangle .
$$

Here $\gamma$ is the ion-electron emission coefficient which depends on the discharge voltage and gas kind; $I_{i}=e v_{i} n_{f} V$ is the ion current which is defined in terms of energy loss by fast electrons in gas ionization in the cathode cavity $V=\left(V_{c}-V_{d}\right)(1-h / 2 D), h$ is the anode height, $D$ is the length and diameter of the cathode cavity; $\delta=(1-\alpha)\left(I / I_{i}\right), \alpha$ is the fraction of fast electrons not involved in ionization; $n_{f}$ and $<\mathrm{v}>$ are the density and average velocity of fast electrons; $v_{i}=n_{g} \sigma_{i} \mathrm{~V}$ is the ionization rate; and $e$ is the electron charge. From (1) we can obtain the condition of the main discharge in the non-self-sustained mode:

$$
u=\frac{P}{P(\gamma+\delta)-1},
$$

which gives a relation between the dimensionless discharge operating voltage $u=e U_{c} / W$, gas pressure $P=\left(p / k T_{e}\right) \sigma_{i} L$, and effective length of the cathode cavity $L=4 V / S_{a} ; W$ is the total electron energy expended in gas ionization. At high pressures $p \gg>\left(k T_{e} / \sigma_{i} L\right)(\gamma+\delta)^{-1}$, the discharge operating voltage does not depend on the effective length of the cathode cavity; the glow discharge condition is defined by the relation $(\gamma+\delta) u=1$, and at $S_{a}<<S_{c}$ it coincides with the relation derived from energy balance and at $\delta=0$ with the relation obtained elsewhere [10, 11, 17].

To determine the spatial distribution of the electron temperature and plasma density and to study the effect of the geometry, size, and material of targets placed in the cathode cavity, we used a hydrodynamic model, which describes the density of charged particles and their average energy as a function of time and space [9].

This model describes the electron charge densities $\left(n_{e}\right)$ and their average energies $\left(n_{\varepsilon}\right)$ as function of the time and space:

$$
\begin{aligned}
& \frac{\partial n_{e, \varepsilon}}{\partial t}+\nabla \vec{\Gamma}_{e, \varepsilon}+\vec{E} \cdot \vec{\Gamma}_{e, \varepsilon}=R_{e, \varepsilon} \\
& \vec{\Gamma}_{e, \varepsilon}=-\left(\mu_{e, \varepsilon} \cdot \vec{E}\right) n_{e, \varepsilon}-D_{e, \varepsilon} \cdot \nabla n_{e, \varepsilon}
\end{aligned}
$$

where $\vec{\Gamma}_{e, \varepsilon}$ are the electron and energy flows, $\mu_{e, \varepsilon}-$ mobility, $\vec{E}$ is electrical field, $D_{e, \varepsilon}$ are diffusion coefficients, $R_{e, \varepsilon}$ are ionization rate and energy loss/gain due to non-elastic collisions. Transport coefficients (discharge coefficients) which are dependent on the electron distribution function are the input data for the fluid dynamics model and calculated by using the BOLSIG + [18]. Modeling of a self-sustained discharge and a discharge with external electron injection is performed without considering the cathode region if the continuity of current on the computation limits is provided. When the current continuity condition is validate the computational domain boundaries, the output parameters for the hydrodynamic model are the discharge characteristics obtained from (1) - (2).

Equation system (1) - (3) allow obtaining interrelations between discharge characteristics: pressure and gas kind, current of auxiliary discharge, energy and ion current, plasma concentration distribution in the hollow cathode and targets placed in it.

Self-sustained mode. In the self-sustained mode, the high voltage of the main discharge is a shortcoming for the hollow cathode, because it leads to intense ion etching of a treated surface. Fig. 2 shows calculated (formula (2)) and experimental dependences $[8,9]$ of the discharge operating voltage on the gas pressure (argon, nitrogen) in the selfsustained mode at different effective lengths of the hollow cathode $L=4 V / S_{a}$; the discharge current is $30 \mathrm{~A}$; the experimental values correspond to $S_{a}=500 \mathrm{~cm}^{2}$. Decreasing the operating pressure of the main discharge causes a substantial increase in discharge operating voltage at a pressure below $0.65 \mathrm{~Pa}$. At these pressures, a large fraction of ionizing electrons whose energy is only partly expended in ionization reaches the anode. The decrease in ionization efficiency is compensated by a corresponding increase in the coefficient of secondary electron emission $\gamma=\gamma(u)$ due to the increase in discharge voltage; in the range of voltages $(200 \ldots 1000) \mathrm{V}, \quad \gamma_{A r}=0.03 \ldots 0.11 \quad$ и $\quad \gamma_{N_{2}}=0.04 \ldots 0.2$, $\left(\sigma_{i A r} / \sigma_{i N_{2}} \approx 1.5\right)$.

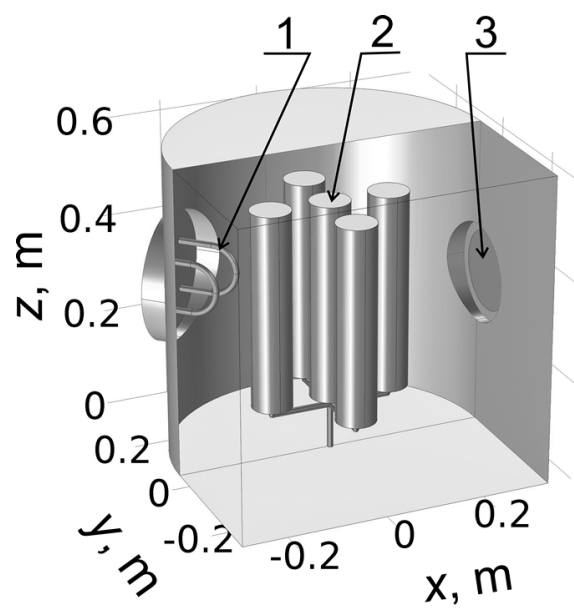

Fig. 1. Hollow cathode model: 1 - anode; 2 - targets; 3 - auxiliary discharge window 
From (2) and Fig. 2 it is seen that decreasing the effective length of the hollow cathode $L=4 V / S_{a}$ (increasing the anode area $S_{a}$ or decreasing the cathode volume due to targets, $\left(V_{c}-V_{d}\right)$ increases the discharge voltage. This owes to capture of fast electrons by the anode, and the self-sustained discharge current is kept at the specified level due to the emission current (the coefficient $\gamma$ ), i.e., due to the voltage in the cathode layer.

The current to the anode is defined by plasma electrons and anode fall. Fig. 3 shows calculated gas pressure dependences of the argon plasma density in the center of the cathode cavity, plasma temperature, and plasma potential in the self-sustained mode at a constant discharge current of $30 \mathrm{~A}$. The discharge current and current density at the cathode are kept constant due to the increase in anode fall with decreasing plasma density $\left(S_{a}=220 \mathrm{~cm}^{2}\right)$.

Non-self-sustained mode. Additional electron injection in to the cathode cavity is through the window of the auxiliary discharge (Fig. 1). As can be seen from (2), the auxiliary discharge shifts the characteristics of the main discharge toward lower voltages and gas pressures. This decreases the energy gained by an electron in the cathode layer of the discharge and the number of its ionization events

Studies of the main discharge operating voltage $U$ depending on the relative volume of targets (Fig. 4) show that in both modes, the voltage $U$ varies slightly at a nitrogen pressure of $p_{N_{2}}>0.65 \mathrm{~Pa}$. In the non-self-sustained mode at an operating pressure of $p_{N_{2}}<0.65 \mathrm{~Pa}$ and discharge voltage of $U>350 \mathrm{~V}$, the main discharge operating voltage can be stabilized by varying the auxiliary discharge current.

The external injection current and plasma potential vs the number of targets $N$ (shaped as cylinders of dimensions $\varnothing 10 \mathrm{~cm} \times 40 \mathrm{~cm}$ ) for $p_{N_{2}}=0.65 \mathrm{~Pa}$ are shown in Fig. 5 . Stabilization of the discharge voltage at $370 \mathrm{~V}$ and ion current density with increasing the number of targets $(N=1 \div 8)$ takes place as the current of additional emission is increased from 9 to $14 \mathrm{~A}$. The plasma potential increases slightly, because the relative volume $\left(V_{c}-V_{d}\right) / V_{c}$ varies in the range of unity up to 0.9 (Fig. 4), the total volume of targets is $(0.25 \div 2) \times 10^{4} \mathrm{~cm}^{3}$, and their relative surface area is $S_{d} / S_{c}=0.071 \div 0.57$.

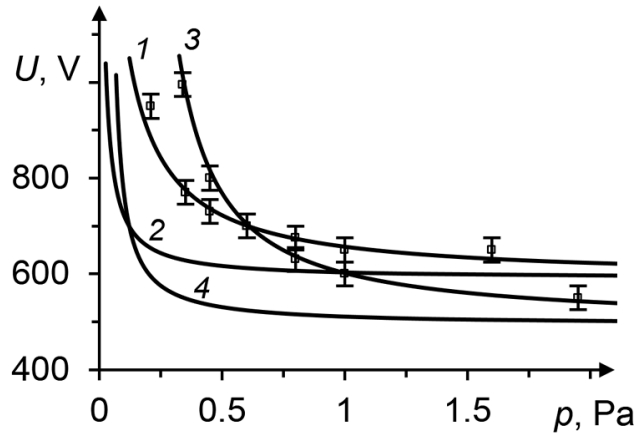

Fig. 2. Discharge operating voltage vs gas pressure in the self-sustained mode for nitrogen $(1,2)$ and argon $(3,4)$ at $L=1.6 \times 10^{3} \mathrm{~cm}(1,3)$ and $L=6.4 \times 10^{3} \mathrm{~cm}(2,4) ; \square-$ experiment, $I=30 \mathrm{~A}$

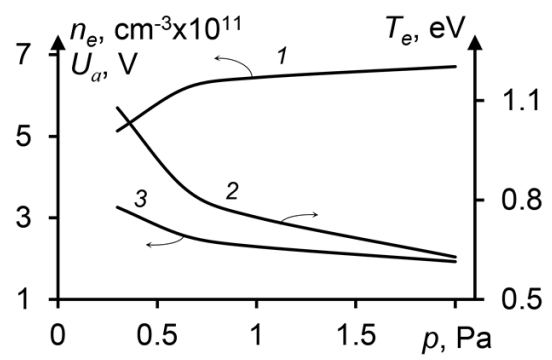

Fig. 3. Calculated pressure dependences of the argon plasma in the selfsustained mode: 1 - plasma density; 2 - plasma temperature; 3 - plasma potential; $S_{a}=220 \mathrm{~cm}^{2}$

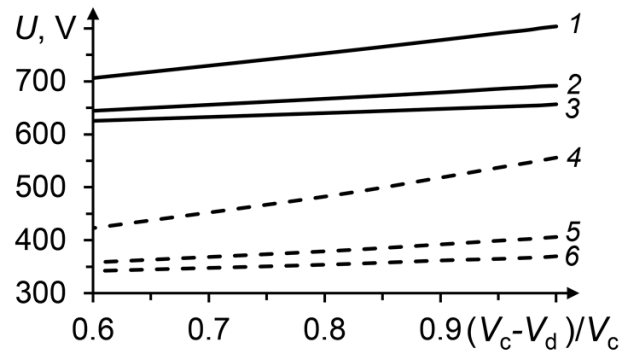

Fig. 4. Discharge voltage in argon vs the relative volume in the self-sustained (solid lines) and non-self-sustained (dashed lines) modes at different pressures $p$ : $0.35(1,4), 0.65(2,5)$, and $1 \mathrm{~Pa}(3,6) ; \delta=0.11$

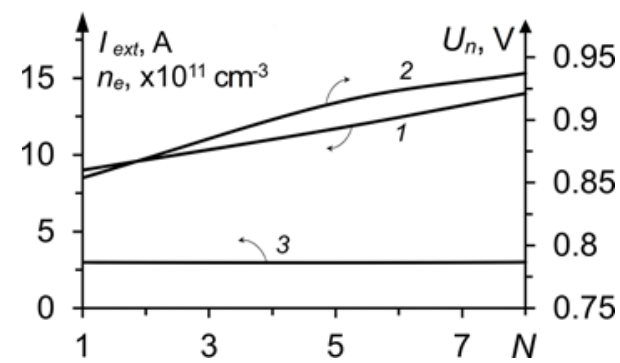

Fig. 5. External injection current (1), plasma density (3), and plasma potential (2) vs the number of targets; nitrogen

Thus, using the theoretical model in which fast electrons are responsible for the birth of charged particles in the hollow cathode plasma, dependences of the discharge operating voltage on the gas pressure and on the geometry of the hollow cathode and treated targets were obtained. It is shown that the discharge operating voltage decreases more than two times due to the current of additional electron injection. It is demonstrated that whatever the surface area and material of treated targets, it is possible to control the ion current density and discharge operating voltage by varying the current of additional electron injection.

Using the hydrodynamic model, we obtained plasma density distributions in the hollow cathode for nitrogen. Fig. 6 shows calculated lines of the plasma density in the non-selfsustained mode at $I_{\text {ext }}=14 \mathrm{~A}$ and $U=370 \mathrm{~V}$ in two sections of the hollow cathode with targets. The pressure gradient due to gas supply through the electron source increases the plasma density gradient in the region of the emission window. It is shown that at a discharge operating voltage of $300-400 \mathrm{~V}$, gas pressure of $0.65 \mathrm{~Pa}$, and plasma temperature of about $1 \mathrm{eV}$, the plasma density for nitrogen and argon reaches $3 \times 10^{11}$ and $6 \times 10^{11} \mathrm{~cm}^{-3}$, respectively. 

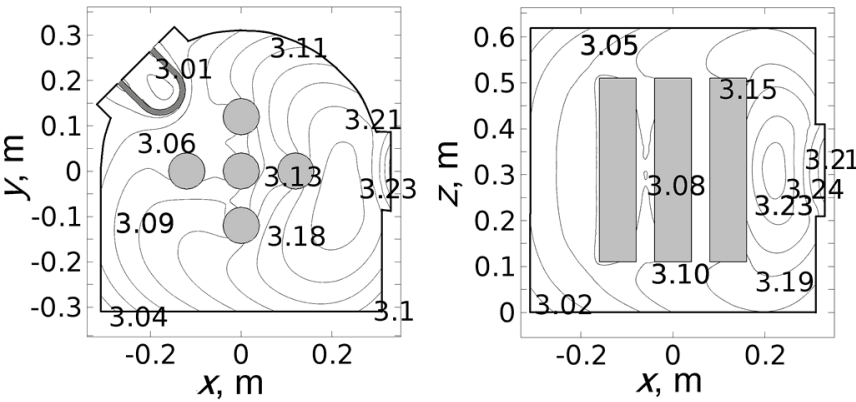

Fig. 6. Plasma density distribution for nitrogen $\left(\mathrm{cm}^{-3} \times 10^{11}\right)$ in the lateral and longitudinal sections of the hollow cathode with targets; non-self-sustained mode

\section{MODELING OF THERMAL PROCESSES}

The properties of the cathode material, configuration of a treated target, and its size can affect the discharge characteristics and the degree of treatment. When treated, a target serves as a cathode; between the plasma and treated target, a space charge layer arises and its electric field provides acceleration of plasma ions. Due to ion bombardment, the target is heated. The heating time depends on the material and dimensions of a treated target. The heat conduction problem with regard to water-cooling of the hollow cathode walls (Fig. 1) was numerically solved for titanium and copper parts of dimensions $\varnothing 5 \div 13 \mathrm{~cm}$ and $20 \div 40 \mathrm{~cm}$. The calculations show that with no shield, the specimen temperature is no greater than $330{ }^{\circ} \mathrm{C}$, which is lower than $530{ }^{\circ} \mathrm{C}$ optimal for nitriding [4, 7].

Active screen surrounds the entire loading parts maker plays a role of the active particles and provides an additional heat radiation parts. The times of heating to $400{ }^{\circ} \mathrm{C}$ for targets of different geometries with an active shield placed at a distance of $2 \mathrm{~cm}$ from the cathode are presented on Fig. 7. The heating time of targets depends on their material and dimensions.

It is seen from Fig. 7 that the difference in heating time for the parts is more than one hour. This defines the difference in nitriding time and hence in nitrogen penetration depth. The energy expended in heating several targets occupying the entire cathode volume can be the same and even lower than the energy expended in heating a single target. This owes to a decrease radiation of targets, which impairs heat removal from them.

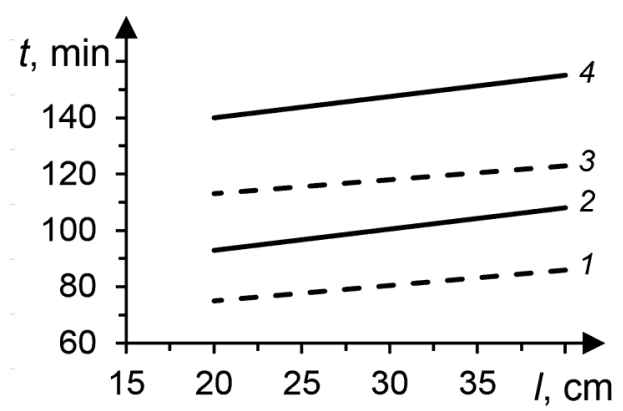

Fig. 7. Heating time vs part length; $1,3-\varnothing=10 \mathrm{~cm} ; 2,4-13 \mathrm{~cm} ; 1,2-$ $\mathrm{Ti} ; 3,4-\mathrm{Fe}$
Hence, the active screen provides uniform heating of targets of different shapes and dimensions to a temperature required for nitriding. The heating time of targets depends on their material and dimensions and decreases with increasing the number of targets due to their mutual thermal radiation. The temperature conditions of treated targets are controlled by the gas pressure and current of the auxiliary discharge.

\section{Modeling of Diffusion-Kinetic Processes}

Experiments show that an increase in nitrogen concentration correlates well with an increase in surface microhardness, and an increase in nitrided layer thickness provides an increase in wear resistance [7]. In this case, thermal diffusion dominates and ion bombardment is secondary and there are two competitive processes: penetration of nitrogen through diffusion and reduction of its depth due to sputtering [19]. The Arrhenius curve characterizes the rate of increase in nitride layer thickness $\ln \left(d^{2} / t\right)$ depending on the temperature $T$ :

$$
d^{2} / t=D_{0} \exp \left(-E_{a} / k T\right)
$$

The linear form of this dependence evidences that the growth of nitride layers is controlled by diffusion of nitrogen atoms, $d$ is the nitride layer thickness, $t$ is the treatment time, $E_{a}$ is the diffusion activation energy determined from experimental data.

The diffusion processes of nitrogen in metal are accompanied by phase changes. The mathematical model of nitriding describes the layer growth kinetics and nitrogen profile concentration at each phase and diffusion zone during ion nitriding of metal. There has been an ongoing effort on the simulation of layer growth kinetics and nitrogen concentration profiles in ion nitriding during the last decades [12-16]. In building the mathematical model of the problem the following assumptions are made: mass balance at the interfaces considers equal specific volumes, flow is one-dimensional, diffusion coefficients are constant at each phase and diffusion zone, the temperature at every point in the specimen is identical during the whole process, the surface erosion and layer growth occur in plane fronts parallel to the specimen surface, evolution of phases takes place under thermodynamical equilibrium conditions.

The diffusion model has the following form:

$$
\frac{\partial C_{i}}{\partial t}=D_{i} \frac{\partial C_{i}^{2}}{\partial x^{2}}
$$

General form of the Fick's first law for the nitrogen diffusion in each phase $(i=1, \ldots, n)$ can be expressed as:

$$
J_{i}=D_{i} \frac{\partial C_{i}(x, t)}{\partial x}
$$

where, $J_{i}$ are nitrogen fluxes, $D_{i}$ are the diffusion coefficients of nitrogen, and $C_{i}=C_{i}(x, t)$ represent the nitrogen concentration at depth $x$ for time $t$ are nitrogen concentrations in the $i$ phases, $\lambda_{i}$ and $x_{i}$ are the thickness and position of $i$ layer $(i=1, \ldots, n)$ respectively. 
The mass conservation rule (Stefan conditions) at the interfaces between layers can be expressed as:

$$
\left[C_{i-1 / i}-C_{i / i-1}\right] \frac{d \xi_{i-1}}{d t}=\left[J_{i-1}-J_{i}\right]_{\xi_{i}}
$$

The model (4) - (6) describes the layer and diffusion zone growth kinetics in the transient stage: layers are completely formed and evolve to a quasi-steady state following a moving boundary diffusion pattern in $C_{i}(x ; t), i=2, \ldots, n$. Equation system (4) - (6) is numerically or analytically solved with Stefan condition executing for interphase boundary.

Nowadays there are several theories explaining the process of nitriding in glow discharge. However, so far researchers have not reached a common opinion on the question of nitrogen saturation mechanism of materials subjected to treating in plasma of gas discharge. One approach to explain the process of saturation, the main role in the process of nitriding in a glow discharge is given to atomic nitrogen, and the intensity of the process is determined by the quantity of atomic nitrogen.

Let consider the problem of iron and steel nitriding. In this case, we have three phases: $\varepsilon$-phase, $\gamma^{\prime}$-phase and $\alpha$-phase $[12,16]$. According to the data from the literature with the assumption of equilibrium in the Fe-N system, the initial and boundary conditions can be expressed as: concentration of nitrogen in $\varepsilon-\mathrm{Fe}_{2-3} \mathrm{~N}$ nitride reaches $11.14-7.71 \mathrm{wt} \%$, concentration of nitrogen in $\gamma^{\prime}$ nitride is in the range of 5.76 to $5.91 \mathrm{wt} \%$. Maximum solubility of nitrogen in $\alpha$-iron, at $590^{\circ} \mathrm{C}$ and at the equilibrium with $\gamma^{\prime}$ nitride, can be considered approximately $C_{\alpha} \approx 0.1 \mathrm{wt} \%$. In this way the nitrogen concentration at interfaces between the $\varepsilon, \gamma^{\prime}$ and $\alpha$ layers can be expressed as: $\mathrm{C}_{\varepsilon / \gamma^{\prime}} \approx 7.71 \mathrm{wt} \%, \quad C_{\gamma^{\prime} \varepsilon} \approx 5.91 \mathrm{wt} \%$, $\mathrm{C} \approx 5.76$ wt $\%$ [16].

Diffusion coefficient of nitrogen in the $\varepsilon, \gamma^{\prime}$ and $\alpha$ phases as a function of temperature was determined elsewhere as:

$$
D_{\varepsilon, \gamma^{\prime}, \alpha}=D_{0, \varepsilon, \gamma^{\prime}, \alpha} \exp \left(\frac{-E_{\varepsilon, \gamma^{\prime}, \alpha}}{R T}\right),
$$

where $\quad D_{0},{ }_{\varepsilon}=2.1 \times 10^{-8} \mathrm{~m}^{2} / \mathrm{s}, \quad D_{0, \gamma^{\prime}}=1.7 \times 10^{-9} \mathrm{~m}^{2} / \mathrm{s}$, $D_{0}, \alpha=6.6 \times 10^{-7} \mathrm{~m}^{2} / \mathrm{s} ; \quad E_{\varepsilon}=93517 \mathrm{~J} / \mathrm{mol}, \quad E_{\gamma^{\prime}}=64000 \mathrm{~J} / \mathrm{mol}$, $E_{\alpha}=77900 \mathrm{~J} / \mathrm{mol}[16] . R=8.314[\mathrm{~J} /(\mathrm{mol} / \mathrm{K})]$ is gas constant. Increasing the nitriding temperature results in increasing the diffusion coefficient of nitrogen in all phases at almost the same rate and leads to a decrease in the ratios of $D_{\alpha} / D_{\varepsilon}$ and $D_{\gamma^{\prime}} / D_{\varepsilon}$, while it increases the ratio $D_{\alpha} / D_{\gamma^{\prime}}$.

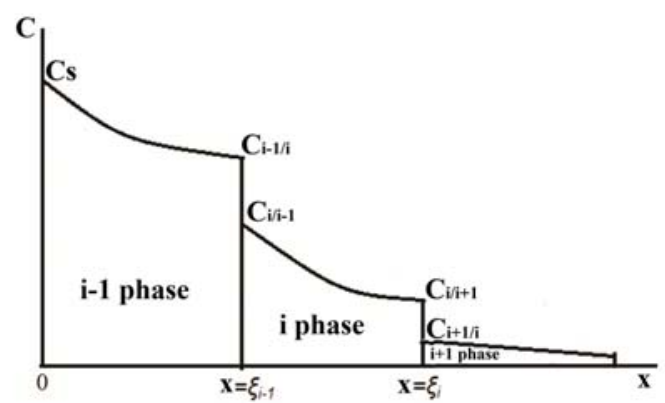

Fig. 8. Prediction of nitrogen distribution in layers
In such a way, the numerical solution of the equation system (4) - (7) with the prescribed initial conditions allow defining the behavior of the layer thicknesses, the nitrogen concentration profiles at each phase and diffusion zone, diffusion zone thicknesses.

\section{COMPARISON WITH EXPERIMENT}

The analysis of the experimental results demonstrates that nitriding of steel 4140 in pure nitrogen for $1-2$ hours results in the increase of microhardness both in the sample surface and depth of the sample (3 times larger) [7]. For steel 4140 the addition of argon or helium in nitrogen does not lead to significant change in microhardness as compared to pure nitrogen. Hardened layer has a thickness of about $100-150 \mu \mathrm{m}$, and the near-surface layer has the maximum hardness, which thickness is $5-10 \mu \mathrm{m}$. The scanning electron microscopy analysis of the microstructure of a cross section steel after nitriding revealed two obviously different layers. Nitride layer has a thickness of $4-10 \mu \mathrm{m}$ depending on the composition of a plasma forming gas mixture, and it is identified as $\mathrm{Fe}_{4} \mathrm{~N}$ [7]. The experimental dependences of the distribution of nitrogen in depth on the plasma-forming gas mixture composition were obtained (Fig. 9).

Fig. 9 shows calculated dependences (equations (4) - (7)) of the distribution of nitrogen in depth in pure iron and steel 4110. In addition to steel iron contains impurities which affect the diffusion coefficients of nitrogen and the concentration of saturated $\alpha$-solution phase $C_{\alpha}$. Moreover, the formation of stable compounds with nitrogen impurities leads to a blurring of the phase boundaries. The numerical experiments for steel account only the change of $C_{\alpha}$ and activation energy $E_{\varepsilon}=$ $95517 \mathrm{~J} / \mathrm{mol}$.

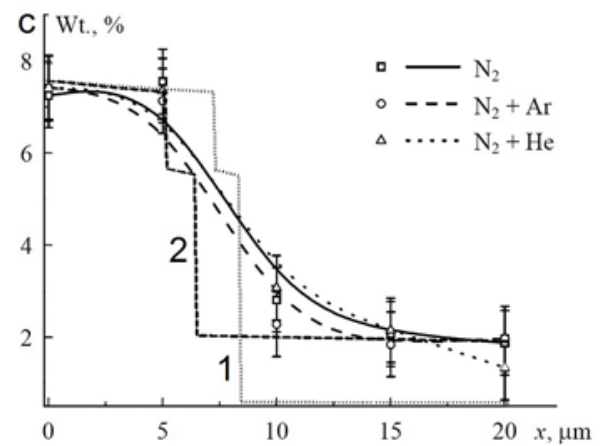

Fig. 9. Experimental [7] and numerical distributions of nitrogen in depth in pure iron (1) and steel (2)

\section{CONCLUSION}

The present work addresses the description of the modeling of processes in low-pressure non-self-sustained glow discharge in a hollow cathode and the plasma chemical nitriding.

This research presents:

- a correctly stated mathematical model determining the relations between the main technological parameters (gas pressure and kind, discharge voltage and current) with the structure of nitrided layer (the nitrogen concentration profiles at each phase and diffusion zone, diffusion zone thicknesses) 
which formed during the nitriding of metal or alloy in low pressure non-self-sustained glow discharge,

- the adequacy and consistency of the mathematical model with the mechanism of low pressure glow discharge generation in the hollow cathode and with the mass transfer mechanism (Fick's Second Law and mass-balance at the interfaces) and the relevant information of the problem (diffusion coefficients and nitrogen solubility limits),

- the study and solution of the mathematical model using obtained analytical condition of discharge ignition, hydrodynamic approximation to determine plasma concentration distribution in the cathode cavity with targets, solution of the thermal conductivity and diffusion-kinetic problem (in MATLAB),

- the qualitative agreement of results of present model with the researches of other authors $[12,16]$ and experimental data [7].

The obtained results allow us to do further research on effective control of layer modification processes with the specified properties.

\section{References}

1. T.A. Panajoti, "Creation of gaseous medium with ultimate saturating power under ion nitriding of the alloys," Fiz. Khim. Obr. Mater., vol. 4, 2003, pp. 70-78.

2. P.M. Schanin, N.N. Koval, I.M. Goncharenko, and S.V. Grigoriev, "Structural steels nitriding in low-pressure gas discharge," Fiz. Khim. Obr. Mater., vol 3, 2001, pp. 16-19.

3. S.R. Hosseini, F. Ashrafizadeh, "Accurate measurement and evaluation of the nitrogen depth profile in plasma nitrided iron,", Vacuum, vol. 83, issue 9, 2009, pp. 1174-1178.

4. L.F. Zagonel, C.A. Figueroa, Jr.R Droppa, F. Alvarez, "Influence of the process temperature on the steel microstructure and hardening in pulsed plasma nitriding," Surf. Coat. Tech. vol. 201, issue 1-2, 2006, pp. 452457.

5. E.V. Berlin, N.N. Koval, L.A. Seidman, "Plasma thermochemical surface treatment of steel parts," Moscow: Tekhnosfera, 2012, p. 462.

6. I.V. Lopatin, Yu.Kh. Akhmadeev, N.N. Koval, P.M. Schanin, V.V. Yakovlev, "Generation of plasma in non-self-sustained glow discharge with hollow cathode modification of materials with particle beams and plasma flows," Proc. of 10th Int. Conf., Tomsk, 19-24 September 2010, pp. 35-38.

7. Yu.H. Akhmadeev, I.V. Lopatin, N.N. Koval, P.M. Schanin, Yu.R. Kolobov, D.S. Vershinin, M.Yu. Smolyakova, "Influence of plasmaforming gas composition on nitriding in non-self-sustained glow discharge with large hollow cathode," 10th International Conference on Modification of Materials with Particle Beams and Plasma Flows, Tomsk, 19-24 September 2010, pp. 228-231.

8. I.V. Lopatin, P.M. Schanin, Y.H. Akhmadeev, S.S. Kovalsky, N.N. Koval, "Self-sustained low pressure glow discharge with a hollow cathode at currents of tens of amperes," Plasma Phys. Rep., vol. 38, issue 7, 2012, pp. 583-589.

9. T.V. Koval, I.V. Lopatin, Nguyen Bao Hung and A.S. Ogorodnikov, "Low pressure discharge characteristics in a large sized hollow cathode," Radiation and Nuclear Techniques in Material Science - Advanced Materials Research., vol. 1084, 2015, pp. 196-199.

10. Yu.D. Korolev, O.B. Frants, N.V. Landl, I.A. Shemyakin, V.G. Geyman, "High-current stages in a low-pressure glow discharge with hollow cathode," IEEE Trans. Plasma Sci., vol. 41, issue 8, 2013, pp. 20872096 .
11. Yu.D. Korolev, K. Frank, "Discharge formation processes and glow-toarc transition in pseudospark switch," IEEE Trans. Plasma Sci., vol. 27 issue 5, 1999, pp.1525-1538.

12. S.R. Hosseini, F. Ashrafizadeh, A. Kermanpur., "Calculation and experimentation of the compound layer thickness in gas and plasma nitriding of iron," Iranian Journal of Science \& Technology, Transaction B: Engineering, vol. 34, issue B5, 2010, pp. 553-566.

13. Mufu Yan, Qingchang Meng, Jihong Yan., "Mathematical models and computer simulation of nitrogen concentration profiles in pulse plasma nitride layers," J. Mater. Sci. Technol., vol. 19, suppl. 1, 2003, pp. 164166.

14. F. Leon Cazares, A. Jimenez Ceniceros, J. Oseguera Pena, and F. Castillo Aranguren, "Modeling surface processes and kinetics of compound layer formation during plasma nitriding of pure iron," Revista Mexicana de F.1sica, vol. 60, 2014, pp. 257-268.

15. J. Bernal, A. Medina, L. Bejar, S. Rangel, A. Juanico, "A diffusion model for coefficient identification during growth of nitrides," International Journal of Mathematical Models and Methods in Applied Sciences, vol. 5 (2), pp. 395-403.

16. V.I. Dimitrov, J.D. Haen, G. Knuyt, C. Quaeyhaegens, L.M. Stals, "Modeling of nitride layer formation during plasma nitriding of iron," Computation Materials Science, vol. 15, 1999, pp. 22-34.

17. A.S. Metel, S.N. Grigoriev, Glow discharge with electrostatic confinement of electrons: physics, engineering, applications. Moscow: MSU IC STANKIN, Janus-K, 2005.

18. G.J.M. Hagelaar, L.C. Pitchford, "Solving the Boltzmann equation to obtain electron transport coefficients and rate coefficients for fluid models," Plasma Sources Sci. Technol., vol. 14, 2005, pp. 722-733.

19. A. Galdikas, T. Moskalioviene, "Modeling of stress induced nitrogen diffusion in nitrided stainless steel," Surf. Coat. Tech. vol. 205, 2011, pp. $3742-3746$. 